Journal of Computer Science 8 (7): 1049-1061, 2012

ISSN 1549-3636

(C) 2012 Science Publications

\title{
Palmprint Recognition using Feature Level Fusion
}

\author{
${ }^{1}$ Gayathri, R. and ${ }^{2} \mathrm{P}$. Ramamoorthy \\ ${ }^{1}$ Department of Electronics and Communication Engineering, \\ Vel Tech Dr. RR and Dr. SR Technical University, Chennai, India \\ ${ }^{2}$ Department of Electronics and Communication Engineering, \\ Sri Shakthi Institute of Engineering Technology, Coimbatore, India
}

\begin{abstract}
Problem statement: Palmprint based biometric method has gained high impact over the other biometric methods due to its ease of acquisition, reliability and high client acceptance. Multiple feature extraction from image gives higher accuracy of the authentication system. Approach: This study presents the palmprint based identification methodology which uses the Gabor wavelet entropy to extract multiple features existing on the palm print, by using a feature level fusion using DempsterShafer theory and are classified using nearest neighbor approach. A feature having the same vector can be grouped together using wavelet transform. A different feature of image using wavelet can be extracted. Some of the features that can be extracted using wavelet entropy consist of contrast, correlation, energy and homogeneity. The features are fused at feature levels. Palmprint matching is then performed by using the nearest neighbor classifier. Results and Conclusion: We selected 100 individuals' left hand palm images; every person is 6 and the total is 600. Later we got every person each palm image as a template (total 100). The remaining 500 were treated as the training samples. The experimental results achieve recognition accuracy of $98.6 \%$ and interesting working point with False Acceptance Rate (FAR) of $=0.03 \%$ and False Rejection Rate (FRR) of $=1.4 \%$ on the publicly available database of The Hong Kong Polytechnic University. Experimental assessment using palmprint image databases clearly validates the efficient recognition performance of the suggested algorithm compared with the conventional palmprint recognition algorithms.
\end{abstract}

Key words: Multifeature, gray level co-occurrence matrix, palmprint, recognition, feature level fusion

\section{INTRODUCTION}

Biometric based recognition is more popular and getting wide acceptance in our information society. Biometrics uses a variety of techniques for identifying a person based on the certain physiological or behavioral attributes. These attributes include fingerprint Jain $e t$ al. $(1997 ;$ 1999) facial features Sonkamble and Thool (2011); Liu and Wechsler (2001), retina and iris patterns Wildes (1997), speech patterns Chou (2000), hand geometry Sanchez-Reillo et al. (2000) and palmprint Zhang and Shu (1999); Duta et al. (2002); You et al. (2002); Kong and Zhang (2002); Chen et al. (2001); Gayathri and Ramamoorthy et al. (2012a; 2012b), Gayathri and Ramamoorthy et al. (2012c); Krishneswari and Arumugam (2012) and Haralick (1979). Biometric features of human being have a unique excellence: It is very ambiguous to remember the lengthy passwords and PIN numbers but biometric passwords are readily available for quick reference for identification. Sometimes they may even lose their cards or they may forget their passwords. It is also unreliable to use token based and knowledge based personal identification. Since those attitudes are not based on any intrinsic characteristics of an individual to make the identification, they cannot able to differentiate between genuine and imposter. For these reasons biometric systems have become predominant in recent years.

Fingerprint identification Jain et al. $(1997 ; 1999)$ is the popular biometric method. There are some situation was finger print not suitable were from the hands of laborers elderly people. Iris based authentication Wildes (1997), has been effectively implemented and was so popular, but it has experienced the uneasiness to capture iris image capturing that requires the user to put their eyes before the capturing device for a longer time. Therefore demand for a novel automatic personal identification system is required.

In recent times, palmprint recognition has acknowledged more attention. The main features of

Corresponding Author: Gayathri. R., Department of Electronics and Communication Engineering, Anna University of Technology, Coimbatore, Tamil Nadu, India Tel: 919884811501 


\section{J. Computer Sci., 8 (7): 1049-1061, 2012}

palmprint contain ridges, wrinkles, principle lines, valleys, minutiae and creases. The area of palmprint is of larger size when compared to fingerprint. And also palmprint contain rich distinctive information than the fingerprint. Since palmprint scanners need to capture a big area, they are bulkier and more expensive than when compared to the other modalities available palm print biometrics approach has several advantages (i) palmprints contain the numerous amount of information when compared to palm hand geometry and fingerprints. (ii) palmprints features varies little over time. (iii) It is having high user acceptability and (iv) even with a low resolution device palmprint is easily captured.

In the field of biometric authentication system, a single modality is not enough to find the similarity between a train image and an input test image. Two or more features can be extracted from the image and can be used for training. The features can be used to form a single vector. These vectors become the training data for the images. We can improve the recognition performance by combining a number of features or combining features. In feature level fusion all the features are extracted from the input image before it is being merged together.

The palmprint biometric is a hand based technology. Palmprint consist of numerable features which are related to the inner surface of a hand. Numerous features of a palm print can be used to uniquely distinguish a person, including (a) Geometry Features: which indicates the shape of the palm which relates geometric features like width length and area. (b) Line Features: it is a vital physiological characteristics of an individual which slightly vary with time. It indicates both location and form of principal lines. (c) Wrinkle Features: It is a thinner and irregular lines found in the palmprint. (d) Delta Point Features: The delta point is defined as the center of a delta-like region in the palmprint. Usually delta points are located in the finger-root region. (e) Minutiae Features: A palm print biometrics comprised of the ridges, valleys, minutiae features to be used as another significant measurement.

Palmprint approaches are broadly classified into five categories: line based subspace-based, local statistical-based, global statistical-based and codingbased approaches. The line-based approaches either develop edge detectors or use the prevailing edge detection approaches to extract palm lines. Subspacebased methods generally involve Principal Component Analysis (PCA), Linear Discriminant Analysis (LDA) and Independent Component Analysis (ICA). Researches also has used discrete cosine to transform
Gabor transforms. A local statistical approach involves such as means and variances are computed for small regions and subdivided regions and assumed as features for investigations. Local statistics are computed based on global statistical features like density, moments and center of gravity from images. Coding methods are gaining features by encoding the filter coefficients using Gabor filters. All the authentication techniques are based on feature extraction, classification and matching.

In this study, we will extract four features of the image. The feature extraction is obtained using the wavelet entropy technique. The wavelet entropy is used for feature extraction of image. The features are: energy, contrast, homogeneity and correlation. These features have same vectors and hence can be fused together. The extraction process starts after the texture analysis of the image.

\section{MATERIALS AND METHODS}

Gabor wavelet delivers finest resolutions in both spatial and frequency domain .It is an optimal basis to extract local features due to its multi resolution and multi orientation properties. Due the above advantage it has been used in many image analysis such as texture classification, face and palmprint recognition.

A general 2-D Gabor function is $\Psi$ (x, y) defined by Eq. 1:

$$
g(x, y)=\frac{1}{2 \pi \sigma_{x} \sigma_{y}} \exp \left[-\frac{1}{2}\left(\frac{x^{2}}{\sigma_{x}^{2}}+\frac{y^{2}}{\sigma_{y}^{2}}\right)+j 2 \pi W_{x}\right]
$$

where, the parameters $\sigma_{x}$ and $\sigma_{y}$ are the standard deviations which represents space constraints of the Gaussian envelope. The parameters $\mathrm{x}$, y represents the centroid localization. The parameters of Gabor filters are set to different scales and orientations for multiple feature extraction. The Fourier transform $\mathrm{G}(\mathrm{u}, \mathrm{v})$ of the Gabor function $\mathrm{g}(\mathrm{x}, \mathrm{y})$ can be written as in Eq. 2:

$\mathrm{G}(\mathrm{u}, \mathrm{v})=\exp \left\{-\frac{1}{2}\left[\frac{(\mathrm{u}-\mathrm{W})^{2}}{\sigma_{\mathrm{u}}^{2}}+\frac{\mathrm{v}^{2}}{\sigma_{\mathrm{v}}^{2}}\right]\right\}$

where, W represents the frequency of the sinusoidal plane and the pair $(\mathrm{u}, \mathrm{v})$ represents the frequency components in the $\mathrm{x}$ and $\mathrm{y}$ direction.where $\sigma_{u}=\frac{1}{2 \pi \alpha_{x}}$ and $\sigma_{v}=\frac{1}{2 \pi \alpha_{x}}$.

By dilations and rotation of mother wavelets $\Psi$ (x, y) one can produce Gabor wavelets. It is explained in the Eq. 3:

$\mathrm{g}_{\mathrm{mn}}(\mathrm{x}, \mathrm{y})=\alpha^{-\mathrm{m}} \mathrm{g}\left(\mathrm{x}^{\prime}, \mathrm{y}^{\prime}\right), \alpha>1$ 
Let us we assume $\mathrm{m}=1, \ldots \ldots \ldots \ldots . \mathrm{S}$ and $\mathrm{n}=$ $1, \ldots \ldots \ldots \ldots . \mathrm{K}$ and $\mathrm{K}$ denotes the dilations and orientations respectively and it is discussed in Eq. 4:

$\mathrm{x}^{\prime}=\alpha^{-\mathrm{m}}(\mathrm{x} \cos \theta+\mathrm{y} \sin \theta)$

$y^{\prime}=\alpha^{-m}(-x \sin \theta+y \cos \theta)$

where, $\theta=\frac{n \pi}{K}$ is the angle. Where $\alpha^{m}$ is the scale factor.

Taking into account the redundant material existing in the filtered image as a result of non orthogonally of Gabor wavelets, thus designed to diminish the redundancy of the Gabor wavelet.

Gabor filter design: let us consider $U_{i}$ and $U_{n}$ represents the lower and the upper desired frequencies. Eq. 5-7 explains the design as follows for computing the filter parameters $\sigma_{\mathrm{u}}$ and $\sigma_{\mathrm{v}}$ Eq. 5-7:

$$
\begin{aligned}
& \alpha=\left(\frac{U_{n}}{U_{1}}\right)^{\frac{-1}{s-1}} \\
& \sigma_{u}=\frac{(\alpha-1) U_{n}}{(\alpha+1) \sqrt{2 \ln 2}} \\
& \sigma_{v}=\tan \left(\frac{\pi}{2 k}\right)\left[U_{n}-2 \ln \left(\frac{\sigma_{u}^{2}}{U_{n}}\right)\right] \\
& {\left[2 \ln 2-\frac{(2 \ln 2)^{2} \sigma_{u}^{2}}{U_{n}^{2}}\right]^{\frac{-1}{2}}}
\end{aligned}
$$

where, $\mathrm{W}=\mathrm{U}_{\mathrm{n}}$. By using Four scale and five orientations we have reached a satisfactory performance in our research.

Gabor feature representation: In order to obtain the Gabor feature we have to convolve the original palm image $\mathrm{X}(\mathrm{x}, \mathrm{y})$ with the Gabor filters. The convolution is explained in Eq. 8 as follows:

$$
\mathrm{W}_{\mathrm{m}, \mathrm{n}}(\mathrm{x}, \mathrm{y})=\iint \mathrm{X}(\mathrm{x}, \mathrm{y}) \mathrm{g}_{\mathrm{mn}}^{*}\left(\mathrm{x}-\mathrm{x}_{0}, \mathrm{y}-\mathrm{y}_{0}\right) \mathrm{dx}_{0} \mathrm{dy}_{0}
$$

where the result due to the scale $\mathrm{S}$ and orientation $\mathrm{K}$ is represented by $\mathrm{W}_{\mathrm{m}, \mathrm{n}}(\mathrm{x}, \mathrm{y})$ Fig. 1 shows the magnitude of the given palmprint image with 20 Gabor filters where $U_{1}=0.04$ and $U_{n}=0.5$, in which four scales and five orientations have been used to obtain the series of Gabor responses. Here we obtained a broad and uniform coverage of the spectrum. Here we are selecting four cycles and five orientations to obtain the palmprint image by using the Gabor wavelet coefficient $\mathrm{W}_{\mathrm{m}, \mathrm{n}}(\mathrm{x}, \mathrm{y})$. where four scales ie $\mathrm{m}=$ $0, \ldots .3$; and 5 orientations ie $n=0, \ldots .4$. By combining the rows the coefficients can be converted into a feature vector $\mathrm{X}_{\mathrm{mn}}$. Eq. 9 represents the discriminating feature vector of an image $I(x, y)$ :

$$
\mathrm{X}=\left[\begin{array}{lll}
\mathrm{X}_{0,0}^{\mathrm{T}} & \mathrm{X}_{0,1}^{\mathrm{T}} \cdots \ldots . \mathrm{X}_{3,7}^{\mathrm{T}}
\end{array}\right]
$$

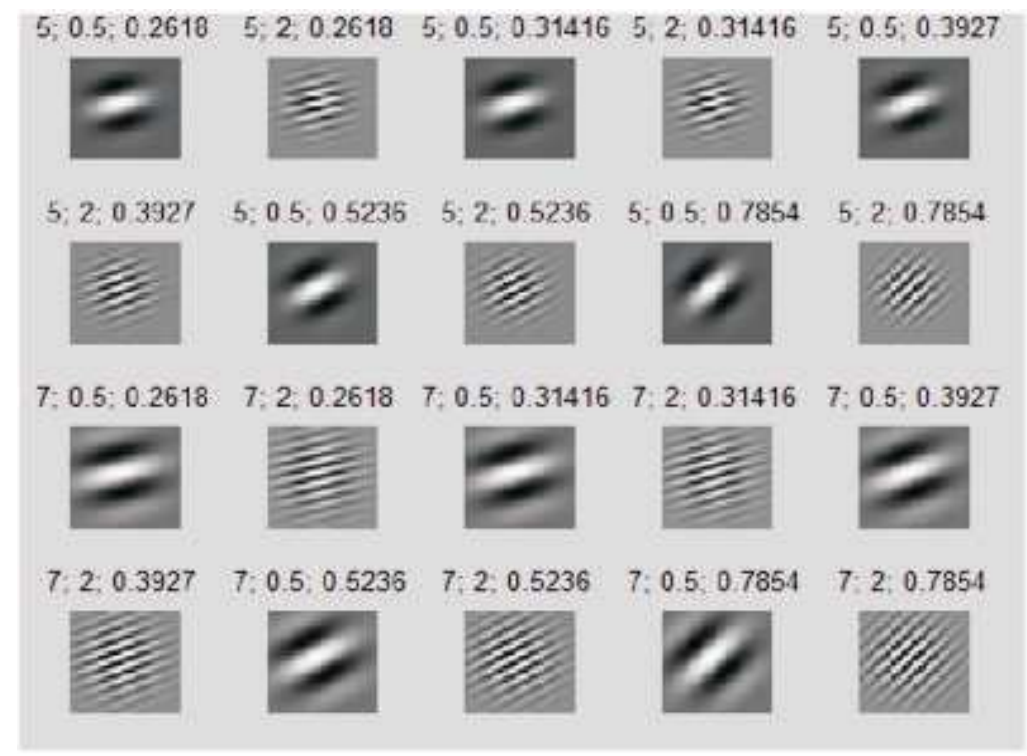

Fig. 1: Representation of Gabor Wavelets (Magnitudes for Spatial frequency $(s)=4$ and Orientation $(k)=5$ ) 


\section{J. Computer Sci., 8 (7): 1049-1061, 2012}

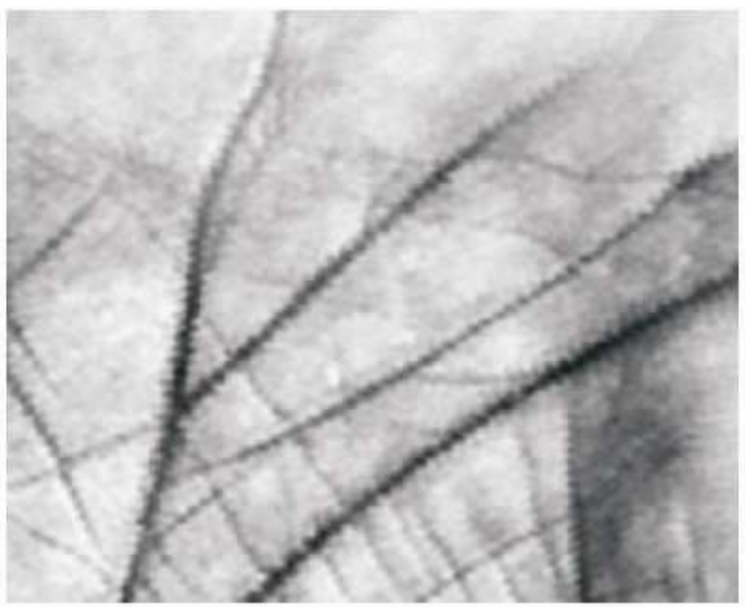

(a)
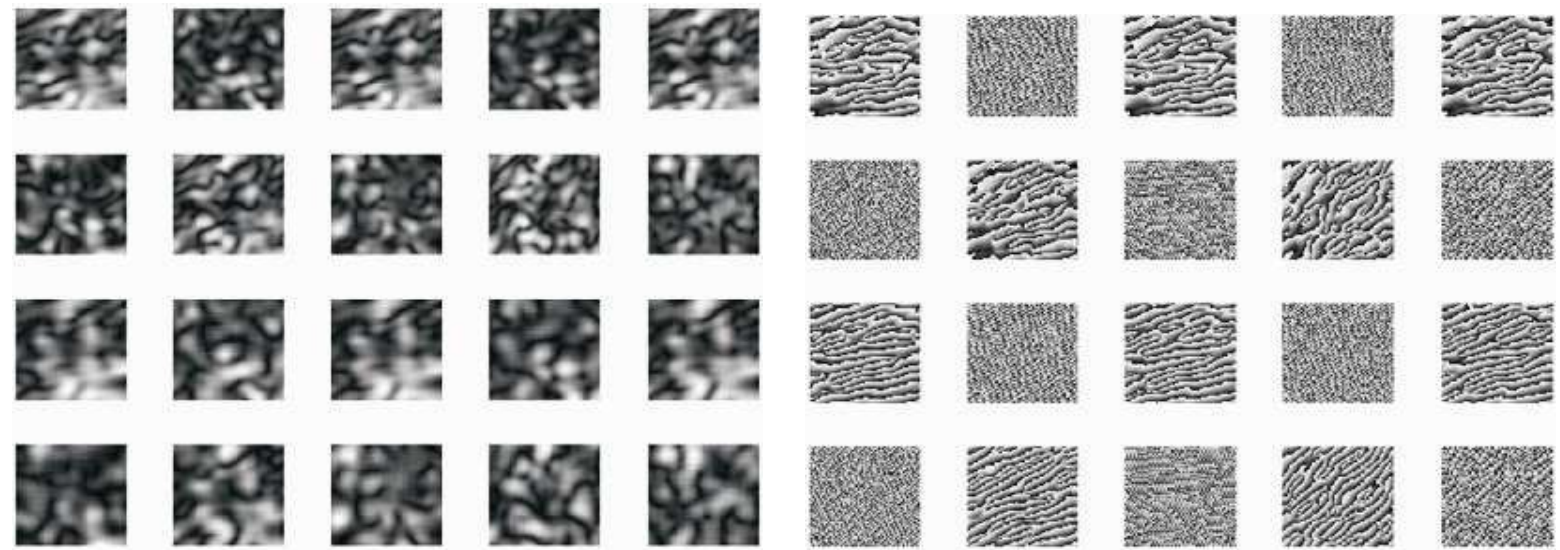

(b)
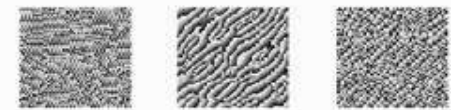

(c)

Fig. 2: Palmprint image response to above Gabor filter (a) original palm image (b) Gabor magnitude Response (c) Gabor phase response. Orientation varies

An image can be represented by the Gabor wavelet by allowing the description of both the spatial frequency and orientation relation. Convoluting the palm image with a complex Gabor filter with 4 spatial frequency and 5 orientations capture the whole frequency spectrum both amplitude and phase as shown in Fig. 1. In Fig. 2, amplitude and phase of Gabor filter responses are shown.

Feature fusion theory of Dempster Shafer: The Dempster Shafer Theory (DST) is a mathematical theory of evidence Shafer (1976). Evidence can be combined from dissimilar sources and arrived at a degree of belief that includes all the available evidence. The theory was first developed by Dempster (1967) and Shafer (1976). The Dempster-Shafer theory is based on the formation of the theory by Dempster and Shafer.
Dempster's rule of combination: Dempsters rule sturdily highlights the agreement between multiple sources and do not take account of all the conflicting evidence through a normalization factor. The combination is calculated from the two sets of masses $\mathrm{m}_{1}$ and $\mathrm{m}_{2}$ as in Eq. 10 and 11 as follows:

$\mathrm{m}_{1,2}(\varnothing)=0$

$\mathrm{m}_{1,2}(\mathrm{~A})=\left(\mathrm{m}_{1} \oplus \mathrm{m}_{2}\right)(\mathrm{A})=\frac{1}{1-\mathrm{K}} \sum_{\mathrm{B} \cap \mathrm{C}=\mathrm{A} \neq \varnothing} \mathrm{m}_{1}(\mathrm{~B}) \mathrm{m}_{2}(\mathrm{C})$

where, $\mathrm{K}=\sum_{\mathrm{B} \cap \mathrm{C}=\varnothing \mathrm{m}_{1}}(\mathrm{~B}) \mathrm{m}_{2}(\mathrm{C})$ and $K$ is a measure of the amount of conflict between the two mass sets $m_{1}$ and $\mathrm{m}_{2}$.

Extraction of entropy features based on GLCM: A co-occurrence matrix is outlined over an image to be 
the spreading of co-occurring values in an identified offset. It can compute the texture of the image by it gray scale values of the image of various extents of color. A co-occurrence matrix $\mathrm{C}$ is explained over an image as given in Eq. 12:

$$
\begin{aligned}
& \mathrm{C} \Delta_{\mathrm{x}}, \Delta_{\mathrm{y}}(\mathrm{i}, \mathrm{j})= \\
& \sum_{\mathrm{p}=1}^{\mathrm{n}} \sum_{\mathrm{q}=1}^{\mathrm{m}}\left\{\begin{array}{cc}
\{1, \text { ifI }(\mathrm{p}, \mathrm{q})=\mathrm{i} \text { and } \mathrm{I}(\mathrm{p}+\Delta \mathrm{x}, \mathrm{q}+\Delta \mathrm{y})=\mathrm{j} \\
0 \quad \text { otherwise }
\end{array}\right.
\end{aligned}
$$

Consider image is a function of $\mathrm{f}(\mathrm{x}, \mathrm{y})$ then the grey level co-occurrence matrix, the probability for grey scale $\mathrm{i}$ and $\mathrm{j}$ (in Eq. 13) and occur at two pixels disjointed by distance $\delta$ and direction $\theta$ :

$$
\begin{gathered}
P(i, j, \delta, \theta)=P(i, j, \Delta x, \Delta y)=P\{f(x, y) \\
=i \operatorname{and} f(x+\Delta x, y+\Delta y=j)
\end{gathered}
$$

By using the gray level co-occurrence matrix texture feature can be calculated. Once the texture features extracted it can be classified and entropy is obtained which is the one of important feature, explained by Eq. 14:

$$
\mathrm{f}_{2}=\sum \sum \mathrm{P}(\mathrm{i}, \mathrm{j}, \delta, \theta) \log _{2} \mathrm{P}(\mathrm{i}, \mathrm{j}, \delta, \theta)
$$

\section{Description:}

- Load the palmprint image

- Selected features such as: energy, contrast, correlation and homogeneity are extracted using wavelet entropy

- The features are merged by wavelet data fusion using Dempster Shafer theory

- Nearest neighborhood algorithm with distance calculation is used for classification of the image

- The test image is classified and the score of matching is calculated and the matched image is taken as the output

Details of feature extraction: The features are extracted from the normalized GLCM. They are as follows.

Contrast: It is to calculate the variation in intensity between a pixel and its adjacent over the entire image. Which is given in Eq. 15:

$$
\sum_{i, j}|i-j|^{2} p(i, j)
$$

Homogeneity: It is to measure the density of the distribution of elements in the GLCM to the GLCM diagonal. Which is explained in Eq. 16:

$$
\sum_{i, j} \frac{p(i, j)}{1+|i, j|}
$$

Energy: It is to compute the sum of squared elements in the GLCM. It is given in Eq. 17:

$\sum_{i, j} p(i, j)^{2}$

Correlation: It is the measure of correlation of the pixel to its neighborhood. It is given in Eq. 18:

$\sum_{i, j} \frac{(i-\mu i)(j-\mu j) p(i, j)}{\sigma_{i} \sigma_{j}}$

Nearest neighborhood classification: The classification is the grouping of the cluster of images between the test image and train image. The mean distance between the centroid of the train image and the test image is calculated. The nearest point is chosen and plots the value which forms a cluster. The distance calculation is based on Euclidean distance weight function. If the value is too far it is not considered.

In 2-D, the Euclidean distance Hu (1962) between $(\mathrm{x} 1, \mathrm{y} 1)$ and $(\mathrm{x} 2, \mathrm{y} 2)$ is given as in Eq. 19:

$$
\sqrt{(x 1-x 2)^{2}+(y 1-y 2)^{2}}=c
$$

Euclidean distance algorithm of classification is non-parametric as their classification is directly dependent on the data of Boiman et al. (2008). The objects are trained according to the data and the test image can be classified using the same process as the object or image was trained.

The following are the significant advantages of the non parametric classifiers:

- It can obviously handle a large number of classes

- It prevents over fitting of the parameters

- No necessity of training and learning stage

The nearest neighbor classifier by Boiman et al. (2008) depend on a distance function between the given points. For all points $\mathrm{x}, \mathrm{y}$ and $\mathrm{z}$, a distance formula $\mathrm{T}$ ( $\mathrm{x}$, $y, z)$ must satisfy the following:

- $\quad$ Symmetry: $\mathrm{T}(\mathrm{x}, \mathrm{y})=\mathrm{T}(\mathrm{y}, \mathrm{x})$

- No negativity: $\mathrm{T}(\mathrm{x}, \mathrm{y}) \geq 0$ 
- $\quad$ Triangle inequality: $\mathrm{T}(\mathrm{x}, \mathrm{y})+\mathrm{T}(\mathrm{y}, \mathrm{z}) \geq \mathrm{T}(\mathrm{x}, \mathrm{z})$

- Reflexivity: $\mathrm{T}(\mathrm{x}, \mathrm{y})=0$ if and only if $\mathrm{x}=\mathrm{y}$

The nearest neighbor classifier is used to find the distance between the input image and the database I already stored image. Let $\mathrm{C}_{11}, \mathrm{C}_{21}, \mathrm{C}_{31} \ldots \mathrm{C}_{\mathrm{k} 1}$ be the $\mathrm{k}$ clusters in the database. The class is found by measuring the distance $T(x)(q), C_{k}$ ) between $x(q)$ and the kth cluster $\mathrm{C}_{\mathrm{k} 1}$. The feature vector with minimum difference is found to be the closest matching vector. It is given by Saradha and Annadurai (2005):

$$
\mathrm{T}\left(\mathrm{x}(\mathrm{q}), \mathrm{C}_{\mathrm{K}}\right)=\min \left\{\|\mathrm{x}(\mathrm{q})-\mathrm{x}\|: \mathrm{x} \in \mathrm{C}_{\mathrm{K}}\right\}
$$

Nearest-neighbor classifiers provide good image classification when the query image is similar to one of the labeled images in its class.

\section{RESULTS AND DISCUSSION}

To evaluate the effectiveness of our proposed palm print biometric authentication scheme, a database containing palmprint samples is required. In this work, we used PolyU palm print database, collected by the biometric research center at The Hong Kong Polytechnic University, is a widely used database in palmprint research. The database contains 7,752 grayscale images' corresponding's to 386 different palms with 20-21 samples for each, in bitmap image format. The experiments are conducted in MATLAB with image processing Toolbox and on a machine with an Intel core 2 Duo CPU processor.

In all the below cases test image and train image are same as follows:

- We selected 5 individuals' left hand palm image every person is 5 and the total is 25.Then we obtained every person's each palm image as a template (total 5). The remaining 20 were treated as training samples. We obtained the Gabor real part and imaginary part accuracy as FRR is $0 \%$ and FAR is $0 \%$ and testing accuracy is $100 \%$

- We selected 25 individuals' left hand palm image every person is 5 and the total is 125 .Then we obtained every person each palm image as a template (total 25). The remaining 100 were treated as the training samples. We obtained the Gabor real part and imaginary part accuracy as FRR is $0 \%$ and FAR is $0 \%$ and accuracy is $100 \%$

- We selected 100 individuals' left hand palm image every person is 6 and the total is 600.Then we get every person's each palm image as a template (total 100). The remaining 500 were treated as the training samples. We obtained the Gabor real part and imaginary part accuracy as FRR is $0 \%$ and FAR is $0 \%$ and accuracy is $100 \%$

First part: We trained the sample and tested it with the same image that was taken as an input test image. Output.

Second part: The train sample is different with the input test image.

In all the cases mention below test image and train image are different:

- We selected 5 individuals' left hand palm image every person is 5 and the total is 25 .Then we obtained every person's each palm image as a template (total 5). The remaining 20 were treated as training samples. We obtained the Gabor real part accuracy as FRR is $1.2 \%$ and FAR is $3 \%$ and testing accuracy is $92 \%$. Gabor Imaginary part accuracy as FRR is $1.2 \%$ FAR is $3 \%$ and testing accuracy is $92 \%$

- We selected 25 individuals' left hand palm image every person is 5 and the total is 125 .Then we obtained every person each palm image as a template (total 25). The remaining 100 were treated as the training samples. We obtained the Gabor real part accuracy as FRR is $0.8 \%$ and FAR is $2.4 \%$ and accuracy is $97.32 \%$. Gabor Imaginary part accuracy as FRR is $0.9 \%$ FAR is $2.4 \%$ and testing accuracy is $97 \%$

Table 1: Accuracy Measures

\begin{tabular}{lllllll}
\hline $\begin{array}{l}\text { No. of } \\
\text { sample }\end{array}$ & \multicolumn{2}{l}{$\begin{array}{l}\text { Gabor real part } \\
\text { accuracy }\end{array}$} & \multicolumn{4}{l}{$\begin{array}{l}\text { Gabor imaginary } \\
\text { part accuracy }\end{array}$} \\
\hline & FRR & FAR & Accuracy & FRR & FAR & Accuracy \\
25 & 1.20 & 3.0 & 92.00 & 1.20 & 3.0 & 92.00 \\
125 & 0.80 & 2.4 & 97.23 & 0.90 & 2.4 & 97.00 \\
600 & 0.03 & 1.4 & 98.6 .0 & 0.04 & 1.4 & 98.32 \\
\hline
\end{tabular}

Table 2: Comparision

\begin{tabular}{lll}
\hline Methods & Database size & Accuracy \\
\hline Proposed Gabor real part & $25 / 5$ & 92.000 \\
Proposed Gabor imaginary part & $25 / 5$ & 92.000 \\
Proposed Gabor real part & $125 / 25$ & 97.230 \\
Proposed Gabor imaginary part & $125 / 25$ & 97.000 \\
Proposed Gabor real part & $600 / 100$ & 98.600 \\
Proposed Gabor imaginary part & $600 / 100$ & 98.320 \\
Canny FSIM Gayathri and & $400 / 100$ & 97.322 \\
Ramamoorthy (2012c) & & \\
Perwitt FSIM Gayathri and & $400 / 100$ & 94.712 \\
Ramamoorthy (2012c) & & \\
Wavelet transforms method & $100 / 50$ & 96.300 \\
\hline
\end{tabular}




\section{J. Computer Sci., 8 (7): 1049-1061, 2012}

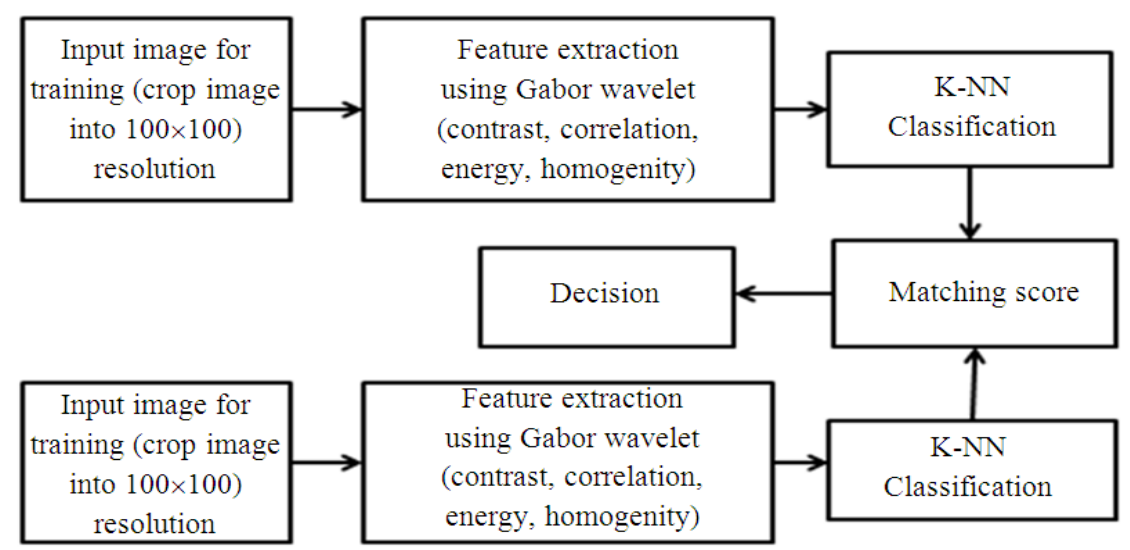

Fig. 3: Block diagram for feature extraction and matching
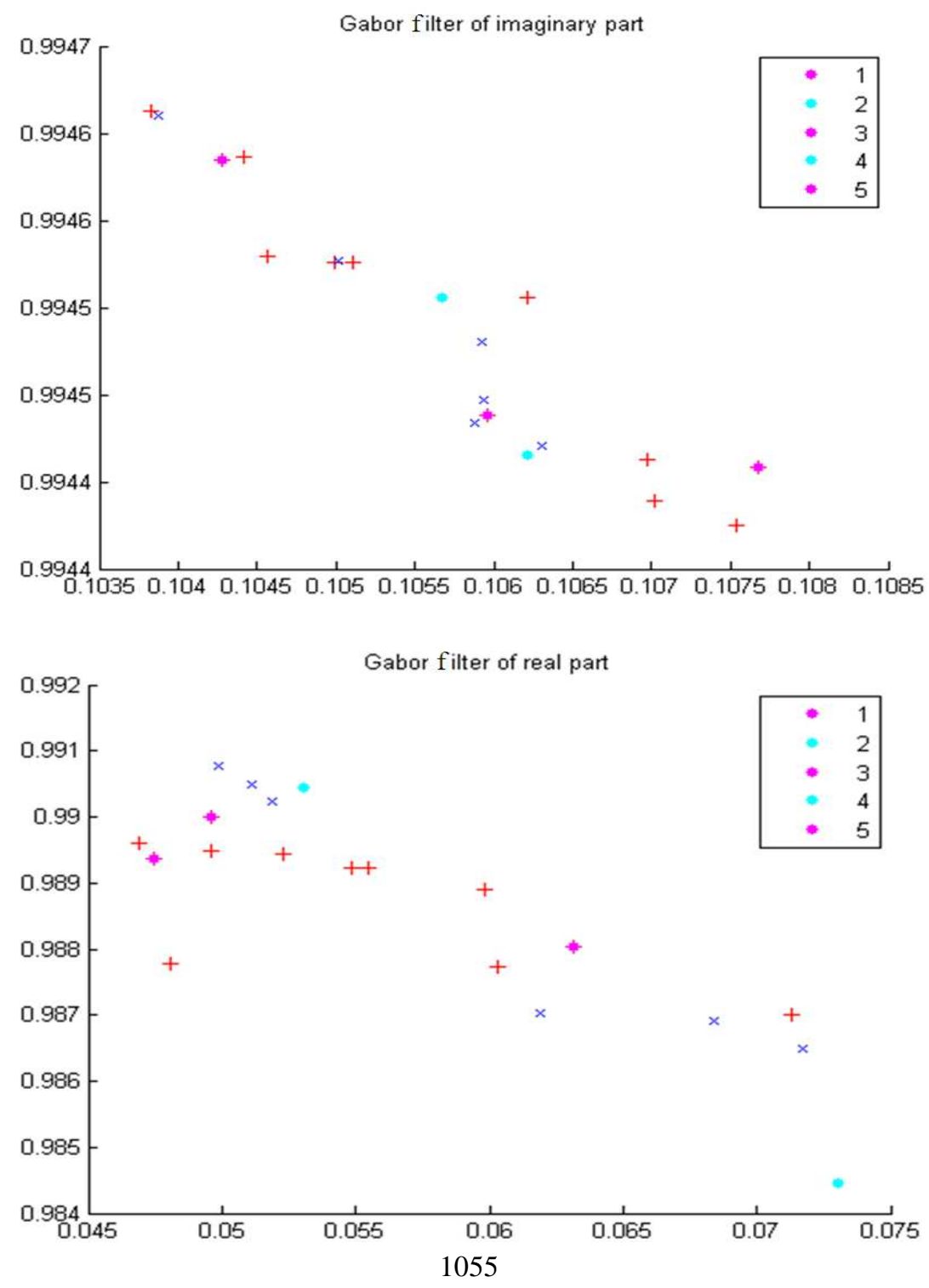
J. Computer Sci., 8 (7): 1049-1061, 2012
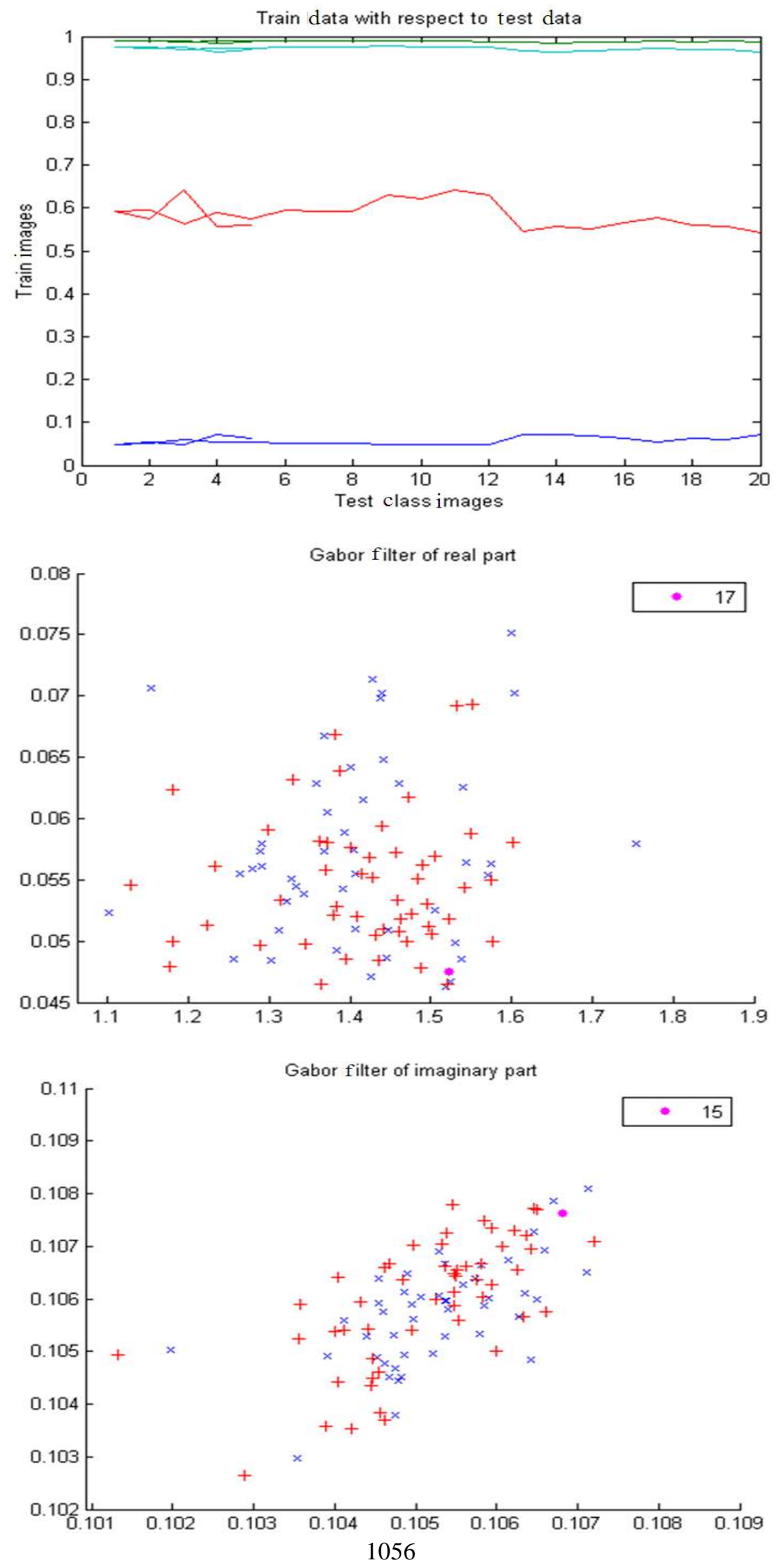
J. Computer Sci., 8 (7): 1049-1061, 2012

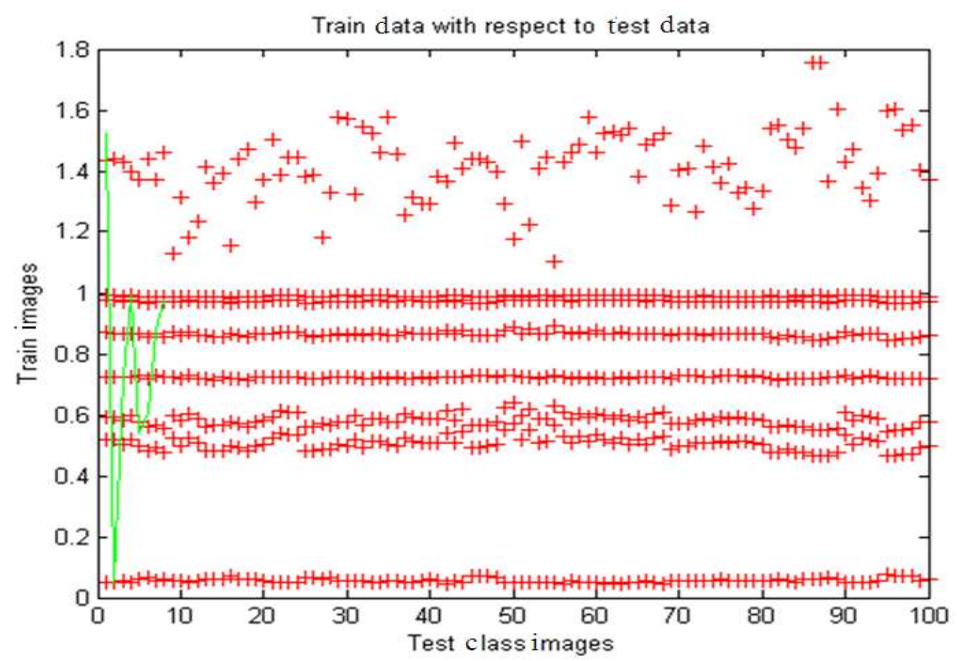

Fig. 4: Result of KNN classification for the palmprint images (i) classification output of 25 samples (test and trained images are the same) (ii) classification output of 125 samples (test and trained images are the same).
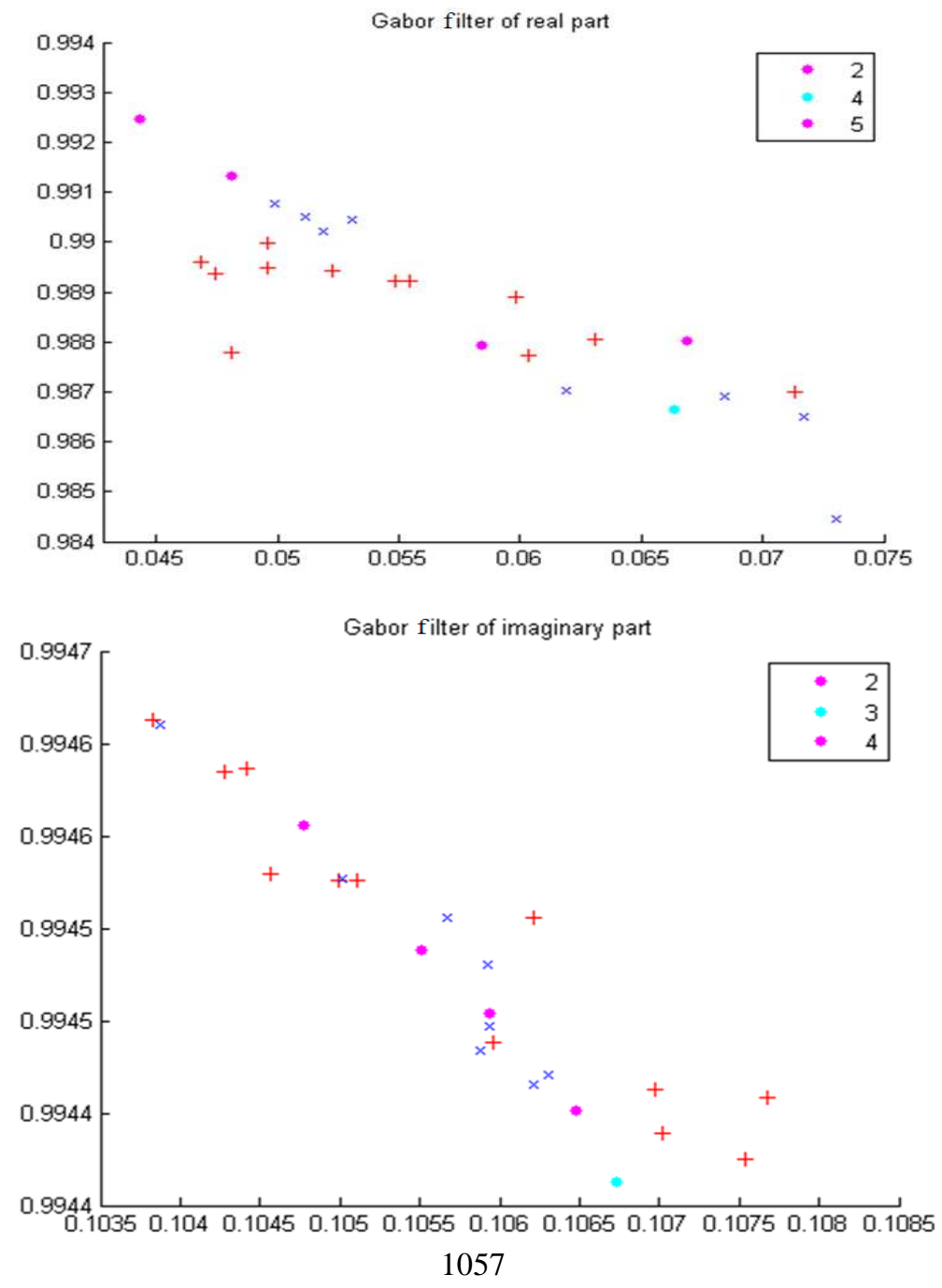
J. Computer Sci., 8 (7): 1049-1061, 2012
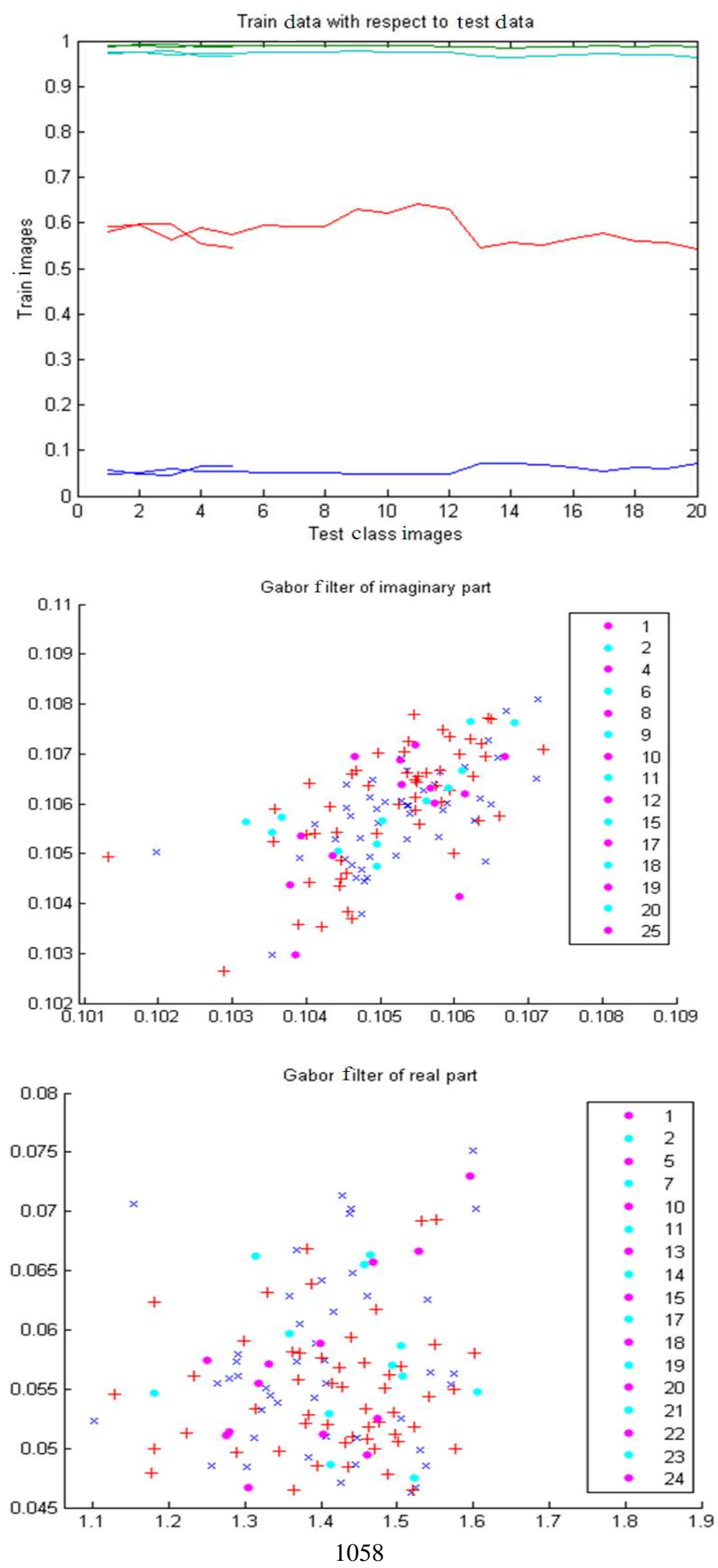
J. Computer Sci., 8 (7): 1049-1061, 2012
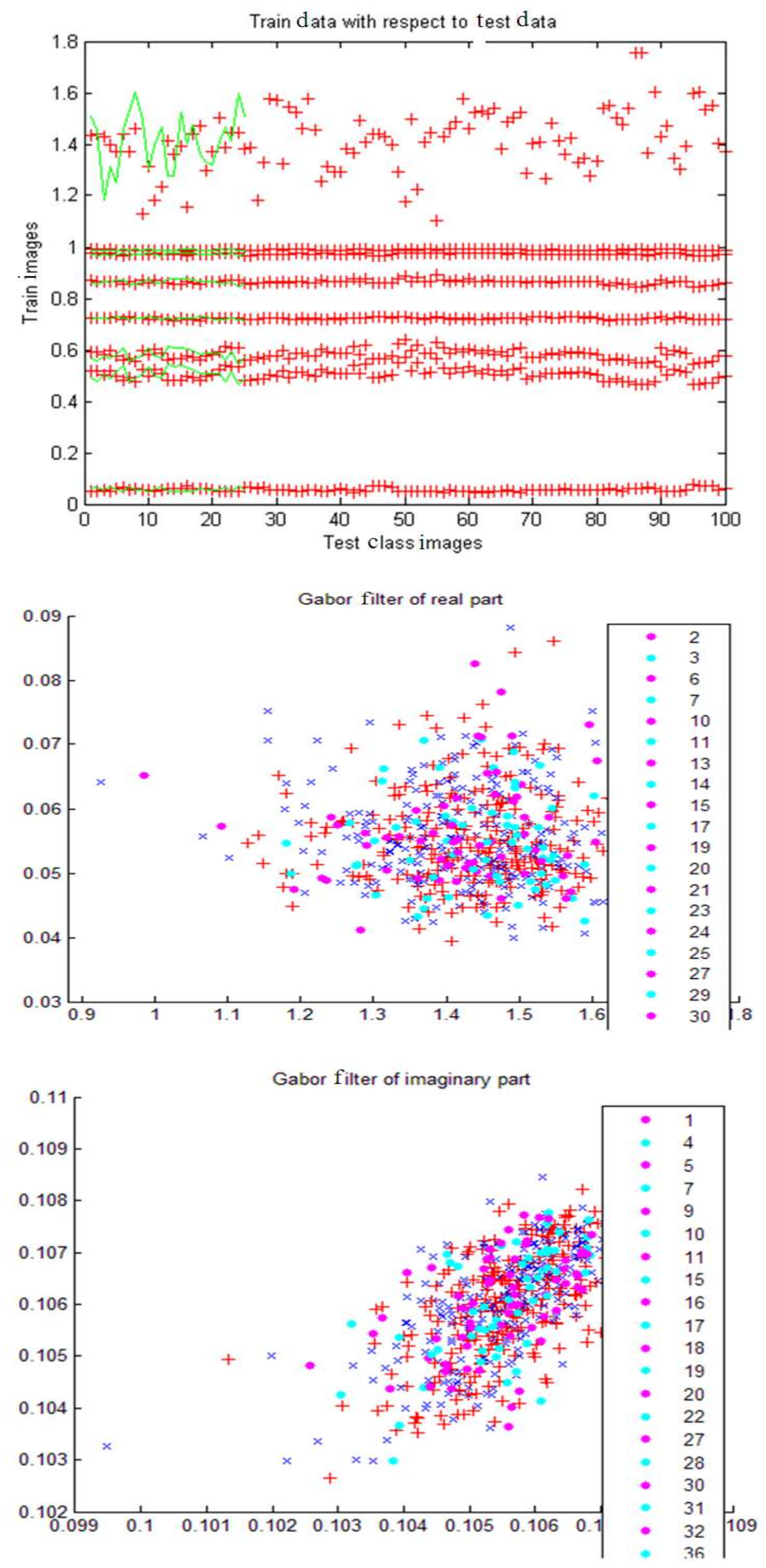

1059 


\section{J. Computer Sci., 8 (7): 1049-1061, 2012}

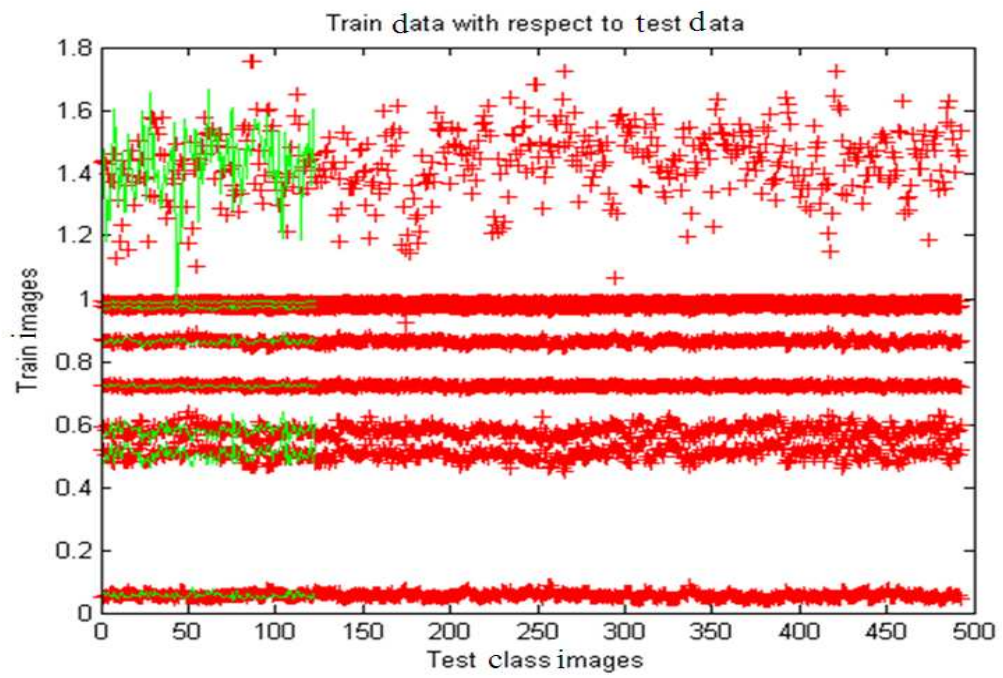

Fig. 5: Result of KNN classification for the palmprint images (iii) Classification output of 25 samples (test and trained images are different) (iv) classification output of 125 samples (test and trained images are different) and (v) classification output of 600 samples (test and trained images are different)

- We selected 100 individuals' left hand palm image every person is 6 and the total is 600 .Then we get every person's each palm image as a template (total 100). The remaining 500 were treated as the training samples. We obtained the Gabor real part accuracy as FRR is $0.03 \%$ and FAR is $1.4 \%$ and accuracy is $98.6 \%$. Gabor Imaginary part accuracy as FRR is $0.04 \%$ FAR is $1.4 \%$ and testing accuracy is $98.32 \%$

From the Fig. 3-5, we analyzed that the image not found are considered as false rejected and the classified class is mismatched with the respective class are considered as false accepted. From Table 2 we can come to a conclusion that the proposed method is efficient.

From the Table 1 classified result, we come to the conclusion that Gabor real part filter value is more accurate compared to Gabor imaginary part filter.

\section{CONCLUSION}

Here the research introduces the well-known Gabor filter transform and its application along with GLCM. The multifeature extraction using wavelet entropy and orientation properties of the Gabor filter makes it a popular method for feature extraction. The reports show the effective fusion of feature using Dempster Shafer theory. The features are fused at feature levels. Palmprint matching is then performed by using the nearest neighbor classifier. The experimental results achieve recognition accuracy of $98.6 \%$ on the publicly available database of The Hong Kong Polytechnic University. Experimental evaluation using palmprint image databases clearly demonstrates the efficient recognition performance of the proposed algorithm.

\section{REFERENCES}

Boiman, O., E. Shechtman and M. Irani, 2008. In defense of nearest-neighbor based image classification. Proceedings of the IEEE Conference on Computer Vision and Pattern Recognition, Jun. 23-28, IEEE Xplore Press, Anchorage, AK., pp: 18. DOI: $10.1109 /$ CVPR.2008.4587598

Chen, J., C. Zhang and G. Rong, 2001. Palmprint recognition using crease. Proceedings of the International Conference Image Processing, Oct. 710, IEEE Xplore Press, Thessaloniki, pp: 234-237. DOI: 10.1109/ICIP.2001.958094

Chou, W., 2000. Discriminant-function-based minimum recognition error rate pattern-recognition approach to speech recognition. Proc. IEEE, 88: 1201-1223. DOI: $10.1109 / 5.880080$

Dempster, A.P., 1967. Upper and lower probabilities induced by a multivalued mapping. Ann. Math. Stat., 38: 325-339. DOI: 10.1214/aoms/1177698950

Duta, N., A.K. Jain and K.V. Mardia, 2002. Matching of palmprints. Patt. Recog. Lett., 23: 477-485. DOI: 10.1016/S0167-8655(01)00179-9

Gayathri, R. and P. Ramamoorthy, 2012a. Automatic personal identification using feature similarity index matching. Am. J. Applied Sci., 9: 678-685. DOI: 10.3844/ajassp.2012.678.685 
Gayathri, R. and P. Ramamoorthy, 2012b. Automatic palmprint identification based on high order Zernike moment. Am. J. Applied Sci., 9: 759-765. DOI: 10.3844/ajassp.2012.759.765

Gayathri, R. and P. Ramamoorthy, 2012c. Multifeature palmprint recognition using feature level fusion. Int. J. Eng. Res. Appli., 2: 1048-1054.

Haralick, R.M., 1979. Statistical and structural approaches to texture. Proc. IEEE, 67: 786-804. DOI: 10.1109/PROC.1979.11328

$\mathrm{Hu}$, M.K., 1962. Visual pattern recognition by moment invariants. IRE Trans. Inform. Theory, 8: 179-187. DOI: 10.1109/TIT.1962.1057692

Jain, A.K., L. Hong, S. Pankanti and R. Bolle, 1997. An identity-authentication system using fingerprints. Proc. IEEE, 85: 1365-1388. DOI: 10.1109/5.628674

Jain, A.K., S. Prabhakar and L. Hong, 1999. A multichannel approach to fingerprint classification. IEEE Trans. Patt. Anal. Mach. Intell., 21: 348-359. DOI: $10.1109 / 34.761265$

Kong, W.K. and D. Zhang, 2002. Palmprint texture analysis based on low-resolution images for personal authentication. Proceedings of the 16th International Conference on Pattern Recognition, Aug. 11-15, IEEE Xplore Press, Canada, pp: 807810. DOI: 10.1109/ICPR.2002.1048142

Krishneswari, K. and S. Arumugam, 2012. Multimodal biometrics using feature fusion. J. Comput. Sci., 8: 431-435. DOI: 10.3844/jcssp.2012.431.435

Liu, C. and H. Wechsler, 2001. A shape-and texturebased enhanced fisher classifier for face recognition. IEEE Trans. Image Process., 10: 598608. DOI: $10.1109 / 83.913594$
Sanchez-Reillo, R., C. Sanchez-Avila and A. GonzalezMarcos, 2000. Biometric identification through hand geometry measurements. IEEE Trans. Patt. Anal. Mach. Intell., 22: 1168-1171. DOI: 10.1109/34.879796

Saradha, S. and A. Annadurai, 2005. A hybrid feature extraction approach for face recognition systems. GVIP J., 5: 23-30.

Shafer, G., 1976. A Mathematical Theory of Evidence. 1st Edn., Princeton University Press, Princeton, N.J., ISBN-10: 0691081751, pp: 297.

Sonkamble, S. and R. Thool, 2011. Efficient feature extraction methodology using wavelet transform for the development of person identification system using Iris biometrics. J. Comput. Sci., 7: 13531357. DOI: $10.3844 /$ jcssp.2011.1353.1357

Wildes, R.P., 1997. Iris recognition: An emerging biometric technology. Proc. IEEE, 85: 1348-1363. DOI: 10.1109/5.628669

You, J., W. Li and D. Zhang, 2002. Hierarchical palmprint identification via multiple feature extraction. Patt. Recog., 35: 847-859. DOI: 10.1016/S0031-3203(01)00100-5

Zhang, D. and W. Shu, 1999. Two novel characteristics in Palmprint verification: Datum point invariance and line feature matching. Patt. Recog., 32: 691702. DOI: 10.1016/S0031-3203(98)00117-4 